\title{
Exploring Twitter as a Game Platform; Strategies and Opportunities for Microblogging-based Games
}

\author{
Kieran Hicks ${ }^{1}$, Kathrin Gerling ${ }^{1}$, Ben Kirman ${ }^{1}$, Conor Linehan ${ }^{2}$, Patrick Dickinson ${ }^{1}$ \\ ${ }^{1}$ University of Lincoln, UK, \{khicks, kgerling, bkirman, pdickinson $\} @$ lincoln.ac.uk \\ ${ }^{2}$ University College Cork, Ireland, conor.linehan@ucc.ie
}

\begin{abstract}
Recent years have seen the massive daily engagement of players with games that are integrated with online social networking sites, such as Facebook. However, few games have successfully created engaging experiences through integration with microblogging websites. In this paper, we explore the opportunities and challenges in using Twitter as a platform for playing games, through the case study of the game Hashtag Dungeon, a dungeon-crawling game that uses Twitter for collaborative creation of game content. Two studies were carried out. A quantitative user study with 32 participants demonstrated that players found the game engaging and rewarding. A follow-up qualitative study with 8 participants suggests that Twitter integration in this game is meaningful, but that there are concerns over the impact of the game on players' Twitter profiles. Based on findings from both studies, we propose strategies for the design of Microblogging-based games, and discuss wider implications of social media integration in games.
\end{abstract}

\section{Author Keywords}

Game Design; Twitter; Evaluation; Design Guidelines; social network games; Commercial

\section{ACM Classification Keywords}

K.8.0 [Personal Computing]: General - Games.

\section{INTRODUCTION}

Social Networking Sites (SNS) have quickly become some of the most popular platforms for online games. For example, at its peak, the game Farmville, which is linked with the Facebook social networking site, was played on a daily basis by hundreds of millions of players [8]. Other games linked with the Facebook platform, such as Candy Crush Saga and Clash of the Clans report tens of millions of daily users. The suitableness of these sites as platforms through which to deliver and play games has been investigated many times from business [15], cultural [42] and usability [30] perspectives. However, not all social

\footnotetext{
Permission to make digital or hard copies of all or part of this work for personal or classroom use is granted without fee provided that copies are not made or distributed for profit or commercial advantage and that copies bear this notice and the full citation on the first page. Copyrights for components of this work owned by others than ACM must be honored. Abstracting with credit is permitted. To copy otherwise, or republish, to post on servers or to redistribute to lists, requires prior specific permission and/or a fee. Request permissions from Permissions@acm.org.
}

CHI PLAY 2015, October 03 - 07, 2015, London, United Kingdom (C) 2015 ACM. ISBN $978-1-4503-3466-2 / 15 / 10 \ldots \$ 15.00$ DOI: http://dx.doi.org/10.1145/2793107.2793119. networking sites are commonly used for the playing of games. Relatively little attention has been paid to the opportunities and challenges provided by using other forms of SNS, such as microblogs or picture messaging services as platforms for games.

The social network Twitter is a very popular example of a "Microblogging" service. In distinction from the privacyconscious and highly-personal focus of services such as Facebook [27], typical use of Twitter involves public sharing of content through small amounts of text and pictures. For example, in Twitter these micro-blog-posts are limited to 140 character "Tweets". Although all content on Twitter is shared publicly with all other users of the service, the development of identifiable social networks is supported through a function that allows users to "follow" another user [25]. Specifically, when a user follows another user, they receive updates from that user in their news feed. Single-topic discussions are also facilitated through the use of searchable \#hashtags. Perhaps surprisingly, given the popularity of the site, and the proliferation of games on other social network sites, Twitter has not become a popular platform for social game development.

In this paper, we explore the opportunities and challenges facing game designers when using Twitter as a platform for playing games. At the center of this exploration is an indepth case study of the game Hashtag Dungeon, a dungeon crawling game that uses Twitter as a means of creating, storing, and promoting game content. We provide an overview of Hashtag Dungeon, and describe how the game leverages Twitter connectivity along with collaborative content creation to enable interaction between players. We report results of two user studies, giving further insights into the play experience of the game. First, we report results from a quantitative user study with 32 participants, demonstrating that players found the game engaging and rewarding. Second, we provide insights into player perceptions of the integration of Twitter with the game, based on a qualitative study with 8 participants. Results show that players generally perceive Twitter integration in Hashtag Dungeon as meaningful - i.e., the integration adds value to their experience rather than barely opening up opportunities for the developer of the game - but that there were some concerns about players' social media profiles and the impact that sharing game-related content. Based on findings from both studies, we propose strategies for the design of Microblogging-based games, and discuss wider implications of social media integration in games. 
As the integration of social network features becomes more prevalent in games, a need for a better understanding of how players perceive and interact through social media integration arises. This paper provides a first enquiry into the integration of Twitter as a central element, offering valuable insights into challenges and opportunities that arise when integrating social media in play.

\section{BACKGROUND}

This section summarizes previous work in the areas of games and social media, along with previous approaches to collaborative content creation in games.

\section{Playing Games on Social Networking Sites}

As social networking sites grew in popularity, developer access to information on users' online social network graphs created new opportunities for game design [19].

\section{Designing games for social networking sites}

Designers have used the information available in players online social network profile in a number of different ways. The most common way of using these data is to promote the game to players' online friends through sharing of game-relevant information. Games invite players to share scores (e.g. Doodle Jump [26], Crossy Road [17]), achievements/progress and game narrative (Sword and Sworcery [6]) with their friends. Although these kinds of integration perhaps offer some social capital to players [43], none can be considered as a game element core to the gameplay experience. Rather, this type of integration gives social network games their 'viral' capacity, as they use the players' own network as an opportunity for promotion of the game [23]. In contrast, games like Parking Wars [29] use the existing real world social relationships between players as a central game dynamic [19, 23]. Players earn points for parking virtual cars on opponents' virtual streets without them noticing. Benefit is gained from knowing when other players will be unable to access the game. Therefore, detailed knowledge of co-players' non-game activities gives players distinct in-game advantages.

Interestingly, Social Network Games have often been criticized for the unimaginative or exploitative use of their players' social networks. For example, Paavilainen et al. [31] identified that 'application spam', referring to the notifications, messages, updates and requests generated by social network games, were identified as a significant cause of frustration among players. This spamming is typical of games that rely on integration with online social networking sites for promotion. Zagal and Björk [47] identify cases where developers have used social network based game mechanics in questionable ways. For example, game developers have been found to enable features without the player's knowledge, and to use emotional and social capital as tools to manipulate player behaviour. Indeed, many players and social network site users have become wary of SNGs due to their tendency to 'spam' and 'pollute' their experience of the social network site.
However, examples such as Parking Wars suggest that, with care, it is possible to use the link between the game and the social network site to produce interesting and engaging game play, without compromising the experience of using the social network site itself. Previous research focuses largely on games played on the Facebook platform, and little is unclear whether the same design considerations exist for other types of social network games.

\section{Designing games for microblogging sites}

There have been several attempts to use microblogging sites as a game platform. 2009's games Spymaster and 140 Mafia integrated Twitter, and used tweets to send out notifications between players based on game activity, but were met with great backlash due to the "spamming" of users' feeds [36]. Japanese game DotWar used Twitter avatars as input for a battle game [37], and LittleCosm was a game driven with real-time sentiment-analysis from Tweets [45]. Witch Potion [1] makes use of the follower system as a friends list for people playing the game. \#WordSnake [10] uses Twitter as a means of playing a word chain game where players Tweet at a certain hashtag. Interestingly, a problem seems to arise when these games become popular: the more players engage with the game, the more games-related Tweets appear on users' feeds, quickly being viewed as spam.

\section{Collaborative Content Creation in Games}

Collaborative content creation is a design feature that has the potential to encourage meaningful interaction between players, thus is interesting to explore in the design of social network games. Collaborative content creation in games refers to the act of multiple users working together to create game content [7], ranging from simple assets (e.g., Guns in Team Fortress 2 [41]) to complex artefacts (e.g., Skywind in The Elder Scrolls V: Skyrim [4]). It allows people to share design ideas, or take advantage of the relative skills of different contributors, and has demonstrated potential for community development [35] and research [21]. An important consequence of content co-creation is that it empowers players to create their own crafted experience of the product, allowing the user to experience independence and self-efficiency [12], gradually shifting ownership from game developers to players [16]. While creating content alone has been demonstrated to be enjoyable [39], collaborative design adds a social element, encouraging players to work together [38], enabling fellowship [18].

We believe that encouraging players to forge a deeper relationship between the game world and social media through collaborative content creation offers a design opportunity to foster engagement, particularly as social networking sites are often used for self-expression [14], an idea which is also present in user generated content in games [34]. Furthermore, collaborative content creation allows us to use Twitter in a manner that is intrinsic to the core mechanics of the game, rather than purely as a marketing tool. 


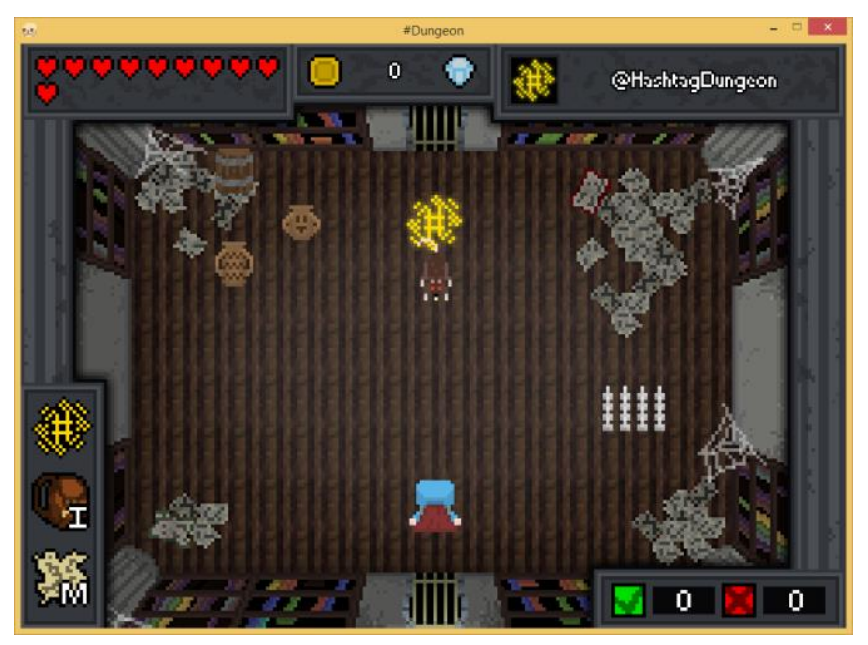

Figure 1. Gameplay view of Hashtag Dungeon.

\section{CASE STUDY: HASHTAG DUNGEON}

In this paper, we examine the use of Twitter as a platform for games through a close case-study of recent commercial game title Hashtag Dungeon. Developed by Hitpoint Games Ltd, a UK based independent game development company, Hashtag Dungeon is a top-down real-time action dungeon crawling game that uses Twitter as a means for creating, storing, and promoting game content. Hashtag Dungeon was implemented in Game Maker Studio [46], and uses the Twitter API [40] to manage Twitter integration. The game is currently available for purchase via itch.io and has recently been greenlit for sale on the Steam digital game distribution service.

\section{Gameplay}

Hashtag Dungeon is, on first glance, a relatively straightforward game where the player must overcome the challenges and perils that lurk inside a dungeon. The player sees the dungeons from a top down perspective and is able to move the avatar in eight directions and can attack by firing projectiles in four directions reminiscent of a twin stick shooter. Each dungeon is comprised of a series of linked rooms, each room may contain enemies for the player to slay, puzzles to solve and traps to avoid. The player takes control of an avatar tasked with clearing all the rooms in a dungeon and then defeating the dungeons boss. When the player enters a room the doors lock preventing them from leaving until that room is clear of enemies, enemies attack the player by either shooting projectiles that the player can move to avoid or by physically attacking the avatar. Enemies can be killed by the player but they have varying health and attack patterns for the player to master, this on top of traps such as spikes that must be avoided and puzzles leads to varied dungeons with a player not knowing what to expect before entering each room. The unique feature of Hashtag Dungeon is that the actual dungeons are generated collaboratively by the player community (Figure 1). The game was originally deployed with an initial set of two dungeons available to players to introduce the basic concepts of the game. The number of dungeons

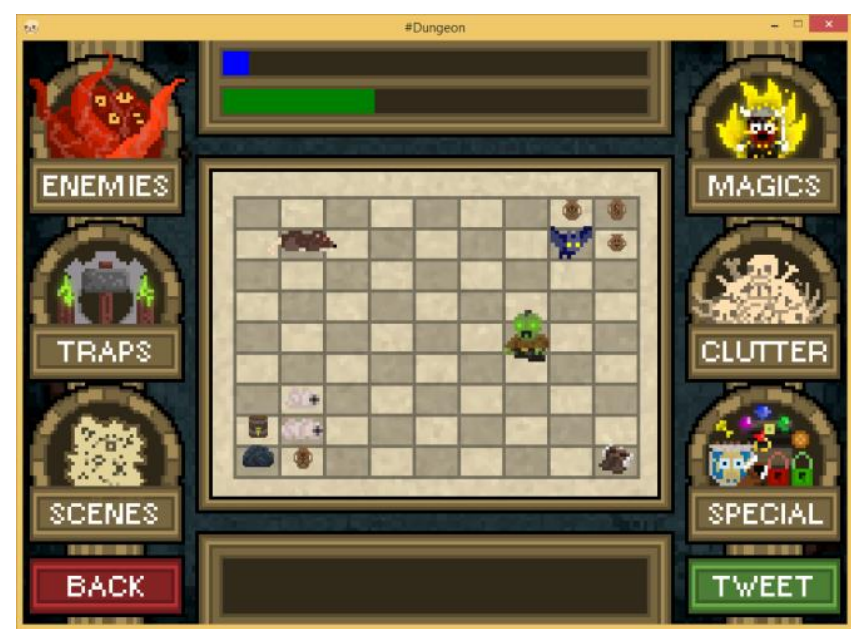

Figure 2. Editor view of Hashtag Dungeon.

available grew quickly as players began using the creative tools and contributed user generated rooms and dungeons.

\section{Collaborative Content Creation}

Hashtag Dungeon allows players to create unique, playable game content. Players can create rooms, which can contain Enemies, Traps, Clutter e.g. Breakable Pots, Items and Scenery. The appearance of the room can also be changed. These rooms are combined to form dungeons based on the room's identifier hashtag (a dungeon is a set of rooms joined together). To support creativity, the game includes an accessible drag-and-drop editing environment (Figure 2). One player uses this environment at a time to specify a design. The player has freedom to add any of the games elements to the room and place them where they wish, however to prevent potential grieving there is a limit on how hard a room can be made. When a player is done designing a room they can they use the editor to Tweet the room with several options such as tweeting it at the dungeon creator or at the official Hashtag Dungeon account once this is done the room becomes available to play.

B In order to promote collaboration in the design of rooms and levels, everything that the user creates is posted automatically to Twitter via the players Twitter account. The intention is to provoke other players who see the new creation in their twitter feed to collaborate on the design (collaborative play; intrinsic integration of OSN), and also to promote curiosity in people who have not yet begun playing the game (social sharing; extrinsic integration of OSN). Thus, if a player wishes to create content for the game they must have and use a Twitter account. When the player has finished creating a room for the game, and wish to make it playable to others, they click on the "Tweet" button in game. This brings up a Twitter window in their default browser. The Tweet is automatically filled out with the details of the room by the game, and the player just needs to click the button to make it public. Content that is posted to twitter is viewable (through searching) by any other player, and, indeed, any other Twitter user. 
Hashtag Dungeon @HashtagDungeon - Feb 7

@HashtagDungeon 0,9/Crypt:85Cauldron:45Witch:Dark60:/

\#HashtagDungeon \#ciarandemise

is $127+0.00$

Hashtag Dungeon@HashtagDungeon.Feb 7

@HashtagDungeon 0,8/Library:43EarthM:63WaterM:Dark60:11C18:70C18: \#HashtagDungeon \#ciarandemise

Figure 3. Room tweets as they are seen on Twitter.

Additionally, there is an option available to players to "hide" their tweet by prepending the tweet with the official Hashtag Dungeon Twitter account. In the mechanics of Twitter, this means that the tweet will only appear on a player's timeline if the reader also follows the Hashtag Dungeon Twitter account. This is a method that allows players to avoid creating "spam" in their Twitter timeline that may be seen by people who are not interested in the game, while still making their Tweet a part of the game's collection of dungeons. Alternatively, players can opt to Tweet it without directing it at the Hashtag Dungeon account, in which case everyone who follows that user will see it on their feed.

\section{Twitter Integration}

The game provides two methods for sharing game content through Twitter. The first involves the automatic sharing, by the game, of details of Dungeons and Rooms created by a player. These Tweets contain the location, dungeon and contents of each room and are not initially human-readable by players (see Figure 3). The first half of the Tweet details where the room is located and the contents of the room. In the example given " 1,2 /Normal" means it's a normal room at position 1,2 in the dungeon. 11B would mean a bat enemy would spawn at that position in the room. Each object that a player can place has a letter or series of letters that are assigned to it. The second half of the Tweet contains 2 Hashtags; the first marks the tweet as a Hashtag Dungeon tweet, and the second hashtag defines which dungeon the room belongs to. In the given example, the dungeon is called "MyDungeon". These two tags help make it quicker for the game server to request and parse data from the Twitter API. The game server only requests Tweets that contain \#HashtagDungeon, which is then stored in a MySQL Database, ordered by the "dungeon name" hashtag. When a player chooses to play a dungeon, that Dungeon name is sent to the server and it returns the tweets that match. By using the server as an intermediary for Twitter ensures the game client only receives the needed amount of data for the dungeon, and is more efficient and faster than when requesting content directly from Twitter.

Players can attach any dungeon name to their room creation tweet. In this way, they can create a room and then decide which of the existing dungeons to attach it to. This allows multiple players to collaboratively construct a sprawling dungeon, through individually creating one room at a time. Also, because any player can attach a room to any of the existing dungeons, it means that no one player can own or

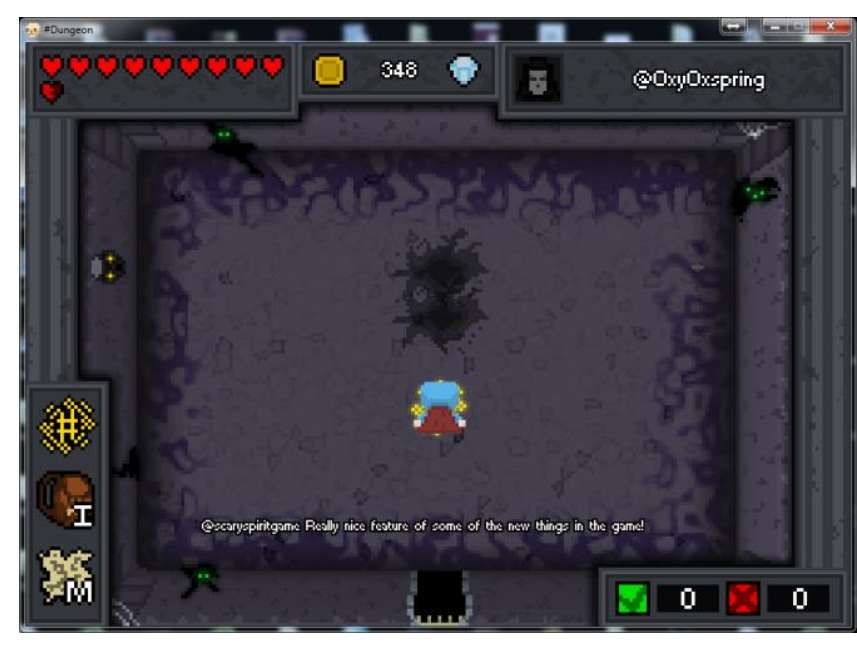

Scaryspirit @scaryspiritgame. Oct 24

tag/0,3/4,5/Really nice feature of some of the new things in the game!/ \#HashtagDungeon \#halloweendungeon \#HashtagDungeon \#hallowee

Figure 4. A player left message as seen in game and on Twitter.

curate a dungeon. They are shifting, amorphous, social constructs. The second method through which a player can share data on Twitter is through leaving a message on the ground in a room. To aid collaboration, Hashtag Dungeon offers players the ability to leave messages (Figure 4) in rooms for other players to read. These messages are simultaneously posted to Twitter; this is done in the same way as posting a room to Twitter and requires the player to have a Twitter account. These messages are not directed at any other accounts and will always show up for followers in their feed. When displayed in-game, the message will show the Twitter handle of the poster next to the message. When a player begins playing Hashtag Dungeon, the game generates the available game content through searching the information that was previously shared on twitter. First, the list of available dungeons is generated from the "dungeon name" hashtags used by players when rooms were created. Secondly, the position of each room in that dungeon is taken from the contents of the related tweets. Lastly the contents of the room e.g. monsters and traps are then spawned based on the related tweets.

The game makes further use of the Twitter API by attaching to every room the Twitter handle and profile picture of the creator of that room. The player can leave feedback on the experience that they had while playing each room using an in-game positive and negative rating system. The total score of each is public and viewable in the room. The creator doesn't receive any notification that a room has been rated.

The design of Hashtag Dungeon orientates itself with the features of the underlying social network. The game is designed to work with normal Twitter usage and expectations, and indeed makes effective use of Twitter's mechanics and dynamics, such as hashtags and follower 
visibility rules. In other words, Twitter is not simply a convenient platform to help promote the game, as many SNGs are criticized for, but instead is integrated completely with the dynamics of Twitter by design. Because of this, it serves as an insightful case study with which to investigate the issues, challenges and opportunities faced in this underexplored design space.

\section{AN ENQUIRY INTO PLAYER PERSPECTIVES ON TWITTER-INTEGRATION IN HASHTAG DUNGEON}

We present results from two user studies examining the playability and player experience of Hashtag Dungeon, as well as exploring perspectives on Twitter integration at various stages in the game's development.

\section{Study 1: Playability and Player Experience}

We carried out an initial evaluation to gain insights into player behavior with respect to co-creation and collaboration along with playability and player experience.

\section{Research Questions}

We aim to answer two main questions related to the experience offered by Hashtag Dungeon, asking whether (1) the game is playable and provides a generally positive player experience, and whether (2) players engage with social features provided by the game in a meaningful way (e.g., by creating dungeons, rating dungeons created by others, and leaving messages within the game).

\section{Measures}

In our study, we apply questionnaires and game metrics to gain insights into player behavior and experience.

Game metrics. In collaboration with the developers, we recorded metrics, with player consent, focused on cocreation, social interaction, and gameplay behaviour. Specifically addressing co-creation and social interaction, we tracked the time players spent exploring dungeons designed by others and the number of rooms cleared, time spent creating new dungeons and the number of dungeons created, and the number of messages sent and rooms rated. Additionally, we tracked number of player deaths and total playing time as an indicator of player performance and level of engagement with Hashtag Dungeon. For a user to see Likes/Dislikes and who created the room a Social tab window had to be brought up. The amount of times each player brought this window up was also recorded.

Questionnaires. We used the Player Experience and Needs Satisfaction (PENS) questionnaire [11] that was developed to assess player experience based on Self-Determination Theory [30]. The questionnaire covers five dimensions, Autonomy, Competence, Immersion, Intuitive Control, and Relatedness. Participants are asked to rate items such as such as "I find the relationships I form in this game fulfilling" and "I feel very capable and effective when playing" on a seven-point Likert scale. The PENS has previously been applied in games research, e.g., $[11,28]$.
Participants and Procedure

We followed a two-step approach to studying player behavior and experience. Due to the nature of the game, which is designed to be played by multiple players simultaneously over a longer period of time, it was not appropriate to carry out a lab study. Initially, we recruited 32 participants via social media. After giving informed consent to the use of game metrics for research and to be contacted for further study, participants were invited to play Hashtag Dungeon online for a period of one week. During this time, we recorded information on their player behavior, including how many dungeons they created, how many dungeons designed by others they engaged with, and whether they decided to use the messaging feature of the game. Following up on this initial engagement with the game, we contacted participants via email and invited them to complete the PENS questionnaire [11] to gain further insights into their experience; 19 of the initial 32 people participated in this step of our research.

Results

We structure our results around the initial questions we raised regarding player experience, co-creation and social interaction in Hashtag Dungeon. Six datasets were excluded from analysis of game metrics because players spent less than five minutes playing the game.

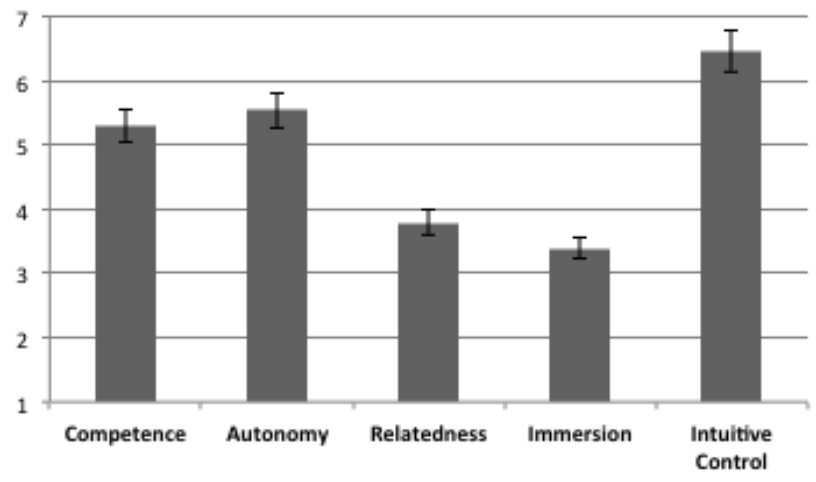

Figure 5. PENS [33] results for Hashtag Dungeon (7=strongly agree, $1=$ =strongly disagree; $\mathrm{CI}=5 \%$ ).

Research Question 1: Does Hashtag Dungeon provide a generally positive player experience? Results of the PENS [33] questionnaire show that Hashtag Dungeon provides above-average player experience regarding competence and autonomy perceived by players along with excellent results for intuitiveness of game controls (see Figure ), suggesting that basic player experience was positive. Game metrics show that that on average, players engaged with the game for 72 minutes (range: 5 minutes to 35 hours; 3:1 split between gameplay and content creation), experiencing an average of 9 deaths and clearing 80 rooms (range: 6 to 3559). Looking at the results for player experience along with these metrics, data suggests that the general design of Hashtag Dungeon was successful, and that key mechanics have potential to appeal to players. In 
contrast to promising findings regarding competence and autonomy, results for immersion and relatedness are below average (see Figure 5). Low scores for immersion could be caused by gameplay requiring players to maintain awareness of events outside of the game world due to Twitter integration; low scores for relatedness are discussed in the context of Research Question 2.

Research Question 2: Do players engage with the social features of Hashtag Dungeon in a meaningful way? Game metrics show that players explored co-creative elements of Hashtag Dungeon, with 25 out of 26 participants collaborating, designing a total of 35 dungeons, including 690 rooms ( $\mathrm{Med}=3$ rooms created by each player). However, there was little engagement with directly social mechanics. Only 7 out of 26 participants used the messaging system, leaving 66 messages in rooms for other players to read. In contrast to this low engagement with the messaging system, there was some engagement with dungeons created by other players, with 31 out of 35 dungeons played at least once $(\mathrm{Med}=10)$, and 14 out of 35 dungeons receiving ratings from other players (26 likes and 10 dislikes). Taking into consideration the low PENS [33] scores for relatedness (Figure 5); explored through the items "I find the relationships I form in this game fulfilling", "I find the relationships I form in this game important", and "I [don't] feel close to other players"), results suggest that while players did engage with content created by others, the game did not encourage the use of directly social mechanics to enable players to connect.

The results of this initial study of player experience provided by Hashtag Dungeon show that the game offered a positive experience in terms of playability and basic game mechanics. However, low use of social mechanics and below-average results for relatedness suggest that further development is necessary to shape Hashtag Dungeon into a game that allows players to connect in a meaningful way. These findings prompted us to follow up with a qualitative study to better understand this notion, allowing us to explore in-depth players' perceptions of social mechanics in general, and Twitter integration in particular.

\section{Study 2: Player Perspectives on Twitter Integration}

Following up on the initial evaluation of player experience, we were interested in examining player perspectives on collaboration and Twitter integration in Hashtag Dungeon. We were specifically interested in those questions that previously emerged in research on the integration of games with social networks, and how to create meaningful instances of social interaction. To this end, we carried out a qualitative enquiry into players' perceptions of the game.

\section{Research Questions}

We were interested in two research questions around Twitter integration and Hashtag Dungeon, (1) asking how participants experienced the game with respect to Twitter connectivity, and (2) how participants perceived and managed social media integration.
Participants and Procedure

We recruited participants through social media, and only included participants with a Twitter account of their own as we were interested in perspectives of Twitter users on the Hashtag Dungeon. As an incentive, participants received a free copy of the game. After giving informed consent, participants received an email with a download code for Hashtag Dungeon, instructions on how to play the game, and two tasks that we asked participants to undertake. Task 1 was to play through a Dungeon of their choice to familiarize themselves with the game. Task 2 was to design a new room for a dungeon and Tweet it out. Afterwards, players were free to continue playing if they wished. Once the two tasks were completed, participants were invited to take part in a structured interview of about 45 minutes. We interviewed 8 participants $(7$ male). The interview was structured around how participants felt about the integration of Twitter in Hashtag Dungeon, and how they manage their own Twitter accounts. There were several questions that each participant was asked with follow up questions based on their responses. At the end of the interview, participants were thanked, given an opportunity to ask questions, and received further information on the study.

\section{Data Collection and Analysis}

To analyze the responses from the interview we followed an inductive Thematic Analysis approach [5]. The responses from the interview were recorded and an analysis was then carried out by the main investigator. This was done following the six phases of Thematic Analysis as proposed by Braun and Clarke [5]. Category codes were applied to responses with a total of 19 categories emerging that fit into 4 themes (see Figure 4).

\section{Results}

From the 299 responses that were analyzed 4 themes arose from the thematic analysis (Figure 6).

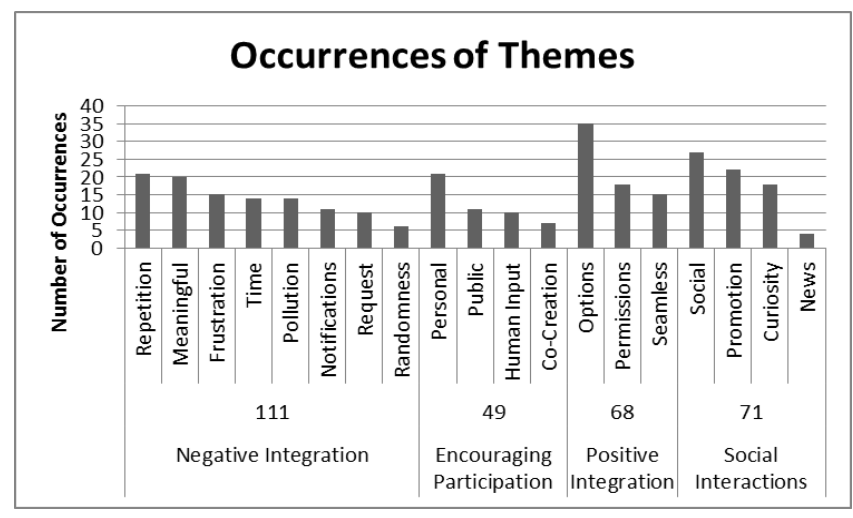

Figure 6. Themes emerging from interviews.

\section{Negative Integration}

The most commonly reoccurring theme (111 times) that emerged from the interviews was the negativity surrounding the integration of social media and games. The biggest cause of negativity that arose from this was in relation to repetition, with participants expressing concern 
that a Twitter-based game would release too much content to their feed, and increase notification frequency. This could be from seeing the same Tweet numerous times, or receiving numerous notifications across devices "tweetdeck is set up to make noise when I get a mention. Also a Phone with the twitter app would buzz and the twitter website will show you have a notification." The meaning of the notification was also a reoccurring theme with negatives with participants stating they would get mentions or notifications that are not of interest to them causing them to feel frustration and that they have "just wasted a few seconds on spam."

The timeframe of the tweets being posted was also a cause of negativity for participants independent of the content. For example it would be fine for fine for a player to Tweet out a dungeon room once an hour, but if it was instead 10 times in 1 minute then it becomes a source of frustration. "promotion of the same thing over a very short time period (e.g. the same picture 30 times in an hour)"

\section{Encouraging Participation}

Participants during the interview were eager to express what features they consider important when using apps that integrate into their social network accounts. Responses that would fit into this theme arose 49 times.

One interesting outcome from this is how each user curates their twitter feed. During the interviews some participants did not mind if an app "spammed" their "if it's just in my feed, it's not that bad." This of course is personal to each user with other participants saying they dislike when applications post on their behalf. We discussed how repetition was met with negative reactions from participants, what emerged from following questions was that participants were eager to be able to add on a "human input" to automated tweets "I feel like you should either add some kind of human input on why you're sharing it".

The human input element also applied when talking about the room Tweets that players tweet when creating content for Hashtag Dungeon. Participants didn't see them as negative or spam due to the fact that it's the players choice to post them "then that's their discretion in question, not the games." The act of co-creation was also something that helped mitigate participants seeing Hashtag Dungeon related tweets as spam "I love to create, and I love seeing others do so too." Seeing other peoples creations pop up on their feed was a positive experience despite the nature of repetitiveness of the tweets.

\section{Positive Integration}

From the responses analyzed the theme of positive aspects arose $(n=68)$, which contained features participants consider to be needed for successful social network integration. The most prevalent of these was the inclusion of options in regards to every aspect associated with the social network. This was shown with all participants praising the ability to choose how and who you post Tweets to "I would tweet to the HashtagDungeon account. I think it's a good thing to include these options". Options also included the option or requirement to authorise an application to post Tweets automatically, participants expressed that while this would help to make integration seamless which was also expressed as being important "doing it without leaving the game would be nice.", concerns were raised about an application forcing you to authorise their account with it "asks me to sign in with facebook or twitter as my ONLY OPTIONS, that is usually enough to get me to stop using it.". Participants expressed that they would like the option to authorise, but would like to still confirm when Tweets are being sent.

\section{Social Interaction}

This theme describes responses $(n=71)$ that would related to how and why participants said they use Twitter. The most recurring theme in this was for Social reasons this included things such as Tweeting, socializing, replying, favoriting and Retweeting "I'll scroll through my feed first thing and retweet and favourite a few tweets, then I'll make a few quick posts".

Participants also stated they used Twitter as a means for promotion of themselves "in the hopes of promoting my writing and my friends' business" this also extended to creating stuff within Hashtag Dungeon participants saw the posting of their tweets an act of promoting their content "you can make dungeons and share them with every player, through one of the busiest social networks is very cool". Promotion was also seen in a bad light with participants showing a dislike for Tweets that exist only to promote/market the game "I'd be more likely to consider something spam if it was in some way advertising / promoting".

Curiosity was also a concept that appeared with users stating how social interactions arose from them posting tweets for the game "it created an conversation topic at the very least!'. Participants attributed this to the cryptic nature of the tweets leading to curiosity "there's an inquisitive element ("what's this Hashtag Dungeon thing?")".

In general, the breadth of themes shows that participants were very aware of issues surrounding social media integration of games and expressed a strong desire for autonomy in terms of managing this integration. In this context, there are a number of aspects to be considered when creating Microblogging-based video games.

\section{CONSIDERATIONS FOR MICROBLOG INTEGRATION}

In this section, we propose four strategies to support the meaningful integration of microblogs that can help inform the work of game designers wishing to integrate social media as game platform. Our considerations specifically address needs of players in the context of social media integration, based on the results of our studies. We focus on elements that can help avoid microblog pollution and encourage meaningful interaction. Many of the strategies 
evolve around features of microblogs (e.g., sharing posts with other users, and receiving notifications), for each strategy, we provide a description along with an example.

\section{Strategy 1: Avoid Empty Notifications}

Social Media can be an extremely content-rich environment. Notifications have emerged as the standard mechanic by which a user is made aware of some actionable or important event within that noisy space. For example, a new follower or friend, a message or a mention. For most users these events are relatively rare. Therefore, it is important to ensure that notifications sent by any applications are similarly meaningful, for example, imply direct interaction of another player or another impactful game event. Our findings from study 2 showed that notifications with a lack of meaning were seen negatively. Although some number of generic notifications may be desirable for design reasons, caution should be used to make sure that the volume is appropriate. Notifications are costly in time and attention for the user who may action each one, in contrast to the general timeline which may be simply skimmed.

For example, in an asynchronous combat game, notifications have more value when they imply action: you have won/lost an important battle, or an enemy army has been spotted advancing on your position, but you have time to organize a defence.

\section{Strategy 2: Introduce Temporal Posting Constraints}

The ability of a game to post content to Twitter should be constrained in order to avoid being seen as "spamming", as our findings showed that the time between posts was significant in avoiding frustration. Constraining the number of tweets seen by any follower to, perhaps 1 or 2 per hour would help ensure that players' Twitter followers are not inundated with lots of updates containing similar content. Note that most platforms, Twitter included, have such a rate-limiting mechanism but only at high volumes. Depending on the design needs an extra limit will likely be desirable. It is interesting that some players considered Hashtag Dungeon as a form of Twitter "bot". Bots are often seen by Twitter users as intrusive on their user experience (e.g. $[9,11])$.

For example, in a farming game, players may act in "bursts", planting a lot of new crops in a short time period. It would be wise to collate these activities into a single summary rather than several similar messages.

\section{Strategy 3: Support Content Curation}

One consistent finding research into the use of online social networks is that users take great care in actively curating their social network profiles $[1,44]$. Users are greatly concerned with presentation of identity, and work hard to ensure content such as photographs, posts and articles shared present a desirable image to other site users. It is important for designers of SNGs to avoid undermining peoples' ability to curate their social network profile. The sharing of links and notifications about playing the game should always be done with consent of the user, and should not be pressurized through exploitative mechanics [47]. This is in line with the findings from study 2 where participants expressed how important it was to have a variety of options on how content is posted.

For example, in an RPG it would be useful to give players editorial control over tweets about their progress. When gaining a level the player can add a personal touch and enrich the shared message to be appropriate for that character's disposition.

\section{Strategy 4: Facilitate Meaningful Sharing}

While the designer is generally able to identify the majority of play moments that are considered important, it is likely that the players have their own understanding of value in the context of each specific game. This will certainly widely vary between the players and as such is difficult for the designer to anticipate and have crafted tweets ready. It is critical that the designer recognizes this and creates opportunities and appropriate tools for players to be able to share any particular aspect of the game in which they find personal meaning. This is likely to be especially important for games that have some space for player creativity (e.g. [22]), where players may take pride in how that creativity is expressed.

For example, in a game where players are able to construct a railway network, they may use the construction tools to draw elaborate shapes and patterns. Although the mechanics aren't explicitly designed for this purpose, the players find meaning in their creativity and wish to share it with their friends, so a tool should exist to support that.

The four strategies described here represent an attempt at making the lessons learnt from our case-study of Hashtag Dungeon more generally useful to the game development community interested in designing games for use on microblogging platforms. Largely they can be summarized as "less is more," with the central warning being that although the tools exist to generate a lot of "noise" about the game through notifications and alerts, and the opportunity for cheap viral promotion is attractive, this should be treated with extreme caution since it has the potential to be received poorly by the players.

\section{DISCUSSION}

This paper presents a first enquiry into Microblog-based play through the case study of Hashtag Dungeon, a dungeon-crawling game that integrates Twitter as cocreative mechanic. Based on results from two user studies, we show that players are generally interested in social media integration in games, but that a number of challenges need to be addressed. We leverage our findings to provide design strategies to support meaningful integration of social media in games. In this section, we focus on Microblogbased co-creation in games, we discuss implications of stereotypes regarding social media integration in games, 
and we discuss how users' need for impression management on social media extends into play.

\section{Co-Creation in Games and Social Media}

The act of co-creation has been proven to increase player engagement while allowing the player to craft their own experience with the product [12]. When including this mechanic with microblog based games our findings show that it helps mitigate the negatives associated with forced posting to microblog sites, with the results from Study 2 showing that when a participant cares about their creation and the game they rationalize that it is different to other spam and are happy to post it public. When a co-creative element is present it also helps players avoid frustration over seeing Tweets about the game on their feed replaced instead with curiosity and intrigue.

\section{Breaking the Stereotype of Social Media Integration in Games as a Marketing Tool}

Dealing with negative player perspectives on social media integration is a key challenge that needs to be overcome when trying to encourage engagement with Microbloggingbased games. Our results show that players were apprehensive to use social features of Hashtag Dungeon, and that they had negative views of games integrated in Social Networking Sites (SNSs). A reason for this might be that many Social Network Games (SNGs) use social media for marketing purposes (i.e., advertising the game to nonplayers by leveraging players' social network) and are viewed as sources of meaningless interaction and spam (e.g., Candy Crush Saga [20]), threatening players' carefully curated social media profiles, an aspect which is also reflected in the "less is more" theme present throughout our design considerations. In this context, an opportunity to foster engagement with Microbloggingbased games is to return to the use of social media as a game platform rather than marketing tool. To this end, it is important to communicate to players how games integrate social media, and to ensure that games enable players to take charge of this integration, giving them authority to decide which information they would like to share. We believe that by focusing on players' benefits rather than those of the provider of the game, engagement can be increased, ultimately creating value for both parties.

\section{Impression Management and Play}

Impression management, maintaining a certain public image, is an important element in the use of social media [23]. Our findings show that this behavior also extends into games, with players being concerned about how engagement in Microblog-based play is represented in their social media profiles. While the logistics of the players' desire to curate their profiles can easily be catered to through careful design of social media integration, an aspect to be considered in terms of game design is how impression management affects player experience. If players constantly monitor how interaction with a game and information sharing with social networks reflects upon themselves, there is significant risk of shifting the focus away from play and breaking immersion. A question that needs to be discussed in this context is whether it is possible to bring the worlds of social media and games together without threatening players' social media identity representation or create a continuous need for identity management, or if the combination of the two will always break the magic circle and have a negative impact on the carefree nature of play [34].

\section{LIMITATIONS AND FUTURE WORK}

There are a number of limitations related to the work presented in this paper. Most importantly, only one game was used in the case study. Additional games need to be considered to provide empirically-grounded design guidelines. Arguably, a purpose built game for microblogging could also help investigate wider issues around such services as game platforms. Additionally, our enquiry into player perceptions of Twitter integration was exploratory and only included a small number of participants. In this context, a larger scale longitudinal study of player experience particularly regarding Twitter integration could provide valuable insights into how players engage with Microblog-based games over a longer period of time. Along these lines, future work should explore implications of facebook games for our work, and pathways of making social network-based games meaningful to people who do not (yet) engage with them, further exploring social media integration of games, and the implications for impression management and play.

\section{CONCLUSION}

Many attempts have been made to implement social network features in games, yet little is known about how players perceive and interact through social media integration. We provide an enquiry into the implementation of Twitter as a game element, and give insights into challenges that need to be addressed to facilitate social media integration in games. Most importantly, our work demonstrates that combining games and social media puts players in the conflicting position of having to manage their social media profile while at the same time addressing ingame challenges, potentially creating tension between one's identity on social media and the role within the game. As this may have implications for player experience, game developers need to be aware of this issue to create games that successfully leverage the full potential of social media integration in games, offer meaningful instances of interaction that empower players to manage their identity, and provide positive experiences, both in-game and on social media.

\section{ACKNOWLEDGMENTS}

We would like to thank all study participants for supporting our research, and we would like to thank Sean Oxspring for his contribution as co-developer of Hashtag Dungeon.

\section{REFERENCES}

1. Alictus (2014). Witch Potion. www.witchpotion.com, last access: 08/04/15. 
2. Back, M. D., Stopfer, J. M., Vazire, S., Gaddis, S., Schmukle, S. C., Egloff, B., \& Gosling, S. D. (2010). Facebook profiles reflect actual personality, not selfidealization. Psychological science.

3. Banks, J. (2002) 'Gamers as Co-Creators: Enlisting the Virtual Audience - A Report from the Net Face', in M. Balnaves, T. O'Regan and J. Sternberg (eds) Mobilising the Audience, pp. 188-212. Brisbane : University of Queensland Press.

4. Bethesda(2011). The Elder Scrolls IV Skyrim. www.elderscrolls.com/, last access: 08/04/15.

5. Braun, V., \& Clarke, V. (2006). Using thematic analysis in psychology. Qualitative research in psychology, 3(2), 77-101.

6. Capybara (2011). Sword \& Sworcery. www.swordandsworcery.com, last access: 08/04/15.

7. Casey, S., Kirman, B., \& Rowland, D. (2007). The gopher game: a social, mobile, locative game with user generated content and peer review. In Proceedings of the international conference on Advances in computer entertainment technology (pp. 9-16). ACM.

8. Cashmore, P.(2010). "FarmVille Surpasses 80 Millions Users" Retrieved 07 April; 2015, from http://mashable.com/2010/02/20/Farmville-80-millionusers/

9. Chu, Z., Gianvecchio, S., Wang, H., \& Jajodia, S. (2012). Detecting automation of twitter accounts: Are you a human, bot, or cyborg?. Dependable and Secure Computing, IEEE Transactions on, 9(6), 811-824.

10. Deen, M., Tieben, R., Oonk, J., Sleegers, T., Kuijk, M. (2012). \#WordSnake.

http://archive.globalgamejam.org/2012/wordsnake, last access 03/07/15

11. Edwards, C., Edwards, A., Spence, P. R., \& Shelton, A. K. (2014). Is that a bot running the social media feed? Testing the differences in perceptions of communication quality for a human agent and a bot agent on Twitter.Computers in Human Behavior, 33, 372-376

12. Füller, J., Rieger, M. and Ihl, C. (2005). The GameCreator: Self-Created Mobile Games On The Internet. In Proceedings of the 2005 DiGRA International Conference: Changing Views: Worlds in Play.

13. Gerling, K.M., Miller, M., Mandryk, R.L., Birk, M., and Smeddinck, J. Effects of Balancing for Physical Abilities on Player Performance, Experience and SelfEsteem in Exergames. In: Proc. of CHI 2014, ACM (2014).

14. Guosong. S., (2009). Understanding the appeal of usergenerated media: a uses and gratification perspective. Internet Research 19.1, 7-25.
15. Hamari, J., \& Järvinen, A. (2011). Building customer relationship through game mechanics in social games. Business, Technological and Social Dimensions of Computer Games: Multidisciplinary Developments. Hershey, PA: IGI Global

16. Harwood, T., \& Garry, T. (2010). 'It's Mine!'Participation and ownership within virtual co-creation environments. Journal of Marketing Management, 26(3-4), 290-301.

17. Hipster Whale(2014). Crossy Road. www.crossyroad.com, last access: 08/04/15.

18. Hunicke, R., LeBlanc, M., \& Zubek, R. (2004). MDA: A formal approach to game design and game research. In Proceedings of the AAAI Workshop on Challenges in Game AI (Vol. 4).

19. Järvinen, A. (2009). Game design for social networks: interaction design for playful dispositions. In Proceedings of the 2009 ACM SIGGRAPH Symposium on Video Games (pp. 95-102). ACM..

20. King(2012). Candy Crush Saga. http://candycrushsaga.com/, last access: 08/04/15.

21. Kirman, B., Casey, S., Lawson, S., \& Rowland, D. (2008). User powered games for research. In Proc: Game Development and Technology Workshop and Conference, Liverpool. ACM.

22. Kirman, B. (2010). Emergence and playfulness in social games. In Proceedings of the 14th International Academic MindTrek Conference: Envisioning Future Media Environments (pp. 71-77). ACM.

23. Kirman, B. (2011). Playful networks: measuring, analysing and understanding the social effects of game design (Doctoral dissertation, University of Lincoln).

24. Krämer, N.C., and Winter, S. Impression Management 2.0. The Relationship of Self-Esteem, Extraversion, Self-Efficacy, and Self-Presentation Within Social Networking Sites. Journal of Media Psychology 20, 3 (2008), 106-116.

25. Kwak, H., Lee, C., Park, H., \& Moon, S. What is Twitter, a social network or a news media?. In Proceedings of the 19th international conference on World wide web (pp. 591-600). ACM. 2010

26. Lima Sky(2009). Doodle Jump. www.limasky.com, last access: 08/04/15.

27. Liu, Y., Gummadi, K. P., Krishnamurthy, B., \& Mislove, A. (2011, November). Analyzing facebook privacy settings: user expectations vs. reality. InProceedings of the 2011 ACM SIGCOMM conference on Internet measurement conference (pp. 61-70). ACM.

28. McEwan, M., Johnson, D., Wyeth, P. \& Blacker, A. Videogame Control Device Impact on the Play Experience. In: Proc. of IE 2012, ACM (2012). 
29. Parking Wars (2007).

https://bbrathwaite.wordpress.com/2007/12/27/faceboo ks-parking-wars-play-dynamics/ , last access: 08/04/15

30. Paavilainen, J. (2012). Design and Evaluation Heuristics for Social Network Games. In Proceedings of Mindrek 2010 conference.

31. Paavilainen, J., Hamari, J., Stenros, J., \& Kinnunen, J. (2013). Social network games: players' perspectives. Simulation \& Gaming, 1046878113514808.

32. Ryan, R.M., and Deci, E.L. Self-determination theory and the facilitation of intrinsic motivation, social development, and well-being. American Psychologist 55 (2000), 68-78.

33. Ryan, R.M., Rigby, C.S., Przybylski, A.K. Motivational pull of video games: A self-determination theory approach. Motivation and Emotion 30, (2006).

34. Salen, K., \& Zimmerman, E. (2004). Rules of play: Game design fundamentals. MIT press.

35. Sarvas, R., Turpeinen, M., Virtanen, P., Hietanen, H. and Herrera, F., (2005). Legal and Organizational Issues in Collaborative User-Created Content. In Proceedings of the 2005 DiGRA International Conference: Changing Views: Worlds in Play.

36. Siegler, M.G. (2009) Spy Vs. Spy: The Spymaster Backlash Begins and Twitter Needs to Fix it. Techcrunch, May $29^{\text {th }}$. Online: http://techcrunch.com/2009/05/29/spy-vs-spy-thespymaster-backlash-begins-and-twitter-needs-to-fix-it/

37. Sipo.jp (2010) Dot War. Online: http://dw.sipo.jp/

38. Sotamaa, O. (2010). When the game is not enough: Motivations and practices among computer game modding culture, Games And Culture, 5, 3, p. 239-255, Scopus, EBSCOhost,

39. Trepte, S., \& Reinecke, L. (2010). Avatar creation and video game enjoyment: Effects of life-satisfaction, game competitiveness, and identification with the avatar. Journal of Media Psychology: Theories, Methods, and Applications, 22(4), 171.

40. Twitter. REST APIs. 2015. Retrieved April 7, 2015 from https://dev.twitter.com/rest/public

41. Valve(2007). Team Fortress 2. www.teamfortress.com/, last access: 08/04/15.

42. Wohn, D. Y., Lampe, C., Wash, R., Ellison, N., \& Vitak, J. (2011). The" S" in Social Network Games: Initiating, Maintaining, and Enhancing Relationships. In System Sciences (HICSS), 2011 44th Hawaii International Conference on (pp. 1-10). IEEE.

43. Wohn, D. Y. (2011). Reciprocity in social network games and generation of social capital. In Annual Meeting of the Association for Education in Journalism and Mass Communication online.
44. Wilson, R. E., Gosling, S. D., \& Graham, L. T. (2012). A review of Facebook research in the social sciences. Perspectives on Psychological Science, 7(3), 203-220.

45. Yongfook, J. (2011) LittleCosm. http://readwrite.com/2010/10/14/littlecosm_a_sentimen t-based_mmorpg_twitter_client, last access: 08/04/15.

46. YoYo Games. GameMaker Studio. 2015. Retrieved April 7, 2015 from https://www.yoyogames.com/studio

47. Zagal, José $P$ and Björk, Staffan and Lewis, Chris (2013) Dark Patterns in the Design of Games. In: Foundations of Digital Games 2013, 16 May 2013, Chania, Crete, Greece. 